\title{
Excitonic fine structure and binding energies of excitonic complexes in single InAs quantum dashes
}

P. Mrowiński, ${ }^{1}$ M. Zieliński, ${ }^{2}$ M. Świderski, ${ }^{2}$ J. Misiewicz, ${ }^{1}$ A. Somers, ${ }^{3}$ J. P. Reithmaier, ${ }^{4}$ S. Höfling, ${ }^{3,5}$ and G. Sęk ${ }^{1}$

${ }^{1}$ Laboratory for Optical Spectroscopy of Nanostructures, Division of Experimental Physics, Faculty of Fundamental Problems of Technology, Wrocław University of Science and Technology, Wrocław, Poland ${ }^{2}$ Institute of Physics, Faculty of Physics, Astronimy and Informatics, Nicolaus Copernicus University, Torun ${ }^{3}$ Technische Physik \& W. C. Röntgen-Center for Complex Material Systems, Universität Würzburg, Germany ${ }^{4}$ Institute of Nanostructure Technologies and Analytics (INA), CINSaT, University of Kassel, Kassel, Germany ${ }^{5}$ SUPA, School of Physics and Astronomy, University of St. Andrews, North Haugh, St. Andrews, United Kingdom

\begin{abstract}
The fundamental electronic and optical properties of elongated InAs nanostructures embedded in quaternary InGaAlAs barrier are investigated by means of high-resolution optical spectroscopy and many-body atomistic tight-binding theory. These wire-like shaped self-assembled nanostructures are known as quantum dashes and are typically formed during the molecular beam epitaxial growth on InP substrates. In this work we study properties of excitonic complexes confined in quantum dashes emitting in a broad spectral range from below 1.2 to $1.55 \mu \mathrm{m}$. We find peculiar trends for the biexciton and negative trion binding energies, with pronounced trion binding in smaller size quantum dashes. These experimental findings are then compared and qualitatively explained by atomistic theory. The theoretical analysis shows a fundamental role of correlation effects for the absolute values of excitonic binding energies. Eventually, we determine the bright exciton fine structure splitting (FSS), where both the experiment and theory predict a broad distribution of the splitting varying from below 50 to almost $180 \mu \mathrm{eV}$. We identify several key factors determining the FSS values in such nanostructures including quantum dash size variation and composition fluctuations.
\end{abstract}

PACS number(s): 78.67.Hc, 73.21.La

\section{INTRODUCTION}

Self-assembled InAs quantum dashes (QDashes) are rather unconventional semiconductor nanostructures with characteristic large in-plane elongation. The potential of QDashes for applications has been demonstrated in various ways, including the utilization as a gain medium for lasers and amplifiers ${ }^{1,2}$ but also as single photon emitters operated both on charged and neutral excitons. ${ }^{3,4}$ What is of special importance and specific for these structures, they are possible to systematically reduce the exciton fine structure splitting (FSS) below the natural linewidth of emission which makes the QDashes prospective as a source of polarization entangled photon pairs from biexciton-exciton cascade. ${ }^{5}$ Such an FSS control seems to be typical for any of QDashes in this material system due to their intrinsic exciton spin properties ${ }^{5}$ which has not been demonstrated to that extent for any other 
quantum-dot-like nanostructures, especially in InP-based nanostructures emitting at telecommunication wavelengths. Examples of such external control have been reported for more symmetric quantum dots of InAsGaAs material system, e.g. by applying electric field to low-strained InAs/InGaAs quantum dots emitting around $1260 \mathrm{~nm},{ }^{6}$ or by magnetic field applied to InAs/GaAs quantum dots emitting below $900 \mathrm{~nm},{ }^{7}$ a reduction of FSS has been obtained. Moreover, QDash exciton bright states exhibit a significant polarization anisotropy of emission, ${ }^{8-10}$ which together with further enhancement by a simple post-growth patterning of dielectric medium can offer an efficient source of linearly polarized single photons, ${ }^{8,10,11}$ which seems to also be quite a unique and application-relevant property of the dashes. All of these overlap with the $2^{\text {nd }}$ or $3^{\text {rd }}$ telecommunication data transmission windows ${ }^{12-14}$ and thus allow considering the QDashes for realization of selected issues in quantum information processing ${ }^{15-17}$ and nanophotonics. ${ }^{7,18-21}$

Quantum dashes are formed in a common self-assembly of molecular beam epitaxy and if they can used asgrown their fabrication is less challenging than nanostructures obtained by more advanced treatment like sitecontrolled methodology $\mathrm{y}^{22-24}$ or strain compensation as for GaAs-based quantum dots for tuning to telecom spectral range, ${ }^{25,26}$ which can hardly reach the $1.55 \mu \mathrm{m}$ range. In spite of the aforementioned technological and device-related perspectives, the fundamental electronic and optical properties of QDashes are still barely known. For such strongly in-plane elongated nanostructures based on $\mathrm{InP}$, there is no systematic investigation both experimental nor theoretical in the context of the influence of morphology and size (energy of emission) on the binding energies of excitonic complexes or the fine structure splitting, or in particular, how significantly the asymmetric potential of the dashes makes them different from the more symmetric InAs-InP-based nanostructures. ${ }^{27-32}$ Such quasi-0D structures can offer unique properties hardly accessible in other systems but also enlighten the understanding of the intermediate regime between strong and weak confinement. Furthermore, it is interesting to learn which factors influence the direct Coulomb interactions, correlations and exchange, and thus affecting the binding energies or the exciton fine structure. This work is supposed to fill this gap leading to a better control of these parameters for next generation of the application-relevant nanostructures using InPbased material system.

The role of morphological details as size and shape, composition profile, on the electronic states (and confinement potential) has been addressed both experimentally and theoretically on numerous kind of III-V material systems ${ }^{33-38}$, including also InAs on $\mathrm{InP}^{28,30}$ but for only slightly asymmetric structures. For example, the importance of correlations, when the size of the nanostructure increases, has been deduced by observing biexciton binding energy increase due to smaller separation between excited hole states. ${ }^{34}$ Other studies have shown also a high sensitivity to relative size and position of electrons and holes wave-functions. ${ }^{39}$

It is well known that the exciton fine structure splitting (FSS) induced by the exchange interaction is very sensitive to the symmetry of the confinement potential, however, the dependence is not always straightforward and differs from system to system due to various factors affecting the confinement potential itself or the electronhole separation. It has been shown, for instance, that even ideal cylindrical symmetry of a nanostructure is not sufficient for obtaining the zero-FSS value, due to built-in intrinsic contribution related to the crystal lattice asymmetry. ${ }^{40}$ The degeneracy itself is lifted by several $\mu \mathrm{eV}$, while in the envelope function theoretical treatment 
it is irrelevant for symmetric structures. Even though, most of the self-assembled quantum dots are far from the cylindrical shape in the plane leading very often to FSS on the order of $100 \mu \mathrm{eV} .{ }^{41,42}$ Such high values that have been observed experimentally, are often missed by theoretical predictions made by $\mathrm{k} \cdot \mathrm{p}{ }^{35}$ or empirical pseudopotential method. ${ }^{43}$ So far, some challenges have been successfully solved by atomistic tight-binding approach $^{42,44}$ which may also be employed for other nontrivial nanostructures. Using atomistic theoretical calculations for deriving excitonic recombination energies that are available experimentally by means of optical measurements, the assessment to the basic structural information has been performed. ${ }^{33,45}$ Additionally, for highly disordered structures the lattice randomness methodology has been examined in order to explain variations in the ordering of the emission spectra within a quantum dot ensemble. ${ }^{46}$

Hereby, we investigated single QDashes both experimentally, using microphotoluminescence, and theoretically by atomistic tight-binding theory combined with the configuration interaction method. Excitonic complexes have been identified in a broad spectral range of emission from 0.8 to above $1.0 \mathrm{eV}(1.2-1.55 \mu \mathrm{m})$ and binding energies of biexciton, charged exciton (trion), and the exciton FSS have been studied. By introducing an elongated geometry with a wedge-shaped cross-section and intermixing of materials, we analyzed Coulomb integrals and correlation corrections as a function of the exciton emission energy. A realistic strongly asymmetric in-plane shape is assumed in order to verify the calculated fine structure splitting dependence with respect to the experimental data.

\section{EXPERIMENTAL}

The investigated structures were grown by a gas-source EIKO molecular-beam epitaxy system using S-doped $\mathrm{InP}(001)$ substrates. A self-assembled InAs layer is deposited at $470^{\circ} \mathrm{C}$ on InGaAlAs barrier lattice matched to InP preceded by InP buffer layer. The same barrier is used to cover the QDashes and then the sample is capped with $10 \mathrm{~nm}$ thick InP. The surface density of QDashes can reach $5 \times 10^{10} \mathrm{~cm}^{-2}$. Significant inhomogeneity of the ensemble combined with etched submicrometer mesa structures allow for probing a relatively small number of QDashes at once and for the observation of single, sharp and well separated emission lines. The typical triangular-shaped cross-section of QDashes has been verified by scanning transmission electron microscopy (STEM) (Figure 1(a)), which also showed that increasing the amount of deposited InAs material enlarges proportionally both the height (approx. 2-4 nm) and width (approx. 10-20 nm) of the nanostructures, leading to a change in the emission wavelength. ${ }^{2,12,13,47}$ Although it is difficult to precisely determine the length scaling according to a scanning electron microscope (SEM) image of uncapped QDash layers (Figure 1(b)), the nominal elongation of more than $50 \mathrm{~nm}$ is observed. Due to the small lattice mismatch of $\sim 3-4 \%,{ }^{48}$ the atom's surface diffusion coefficient anisotropy is more pronounced and then the epitaxially formed nanostructures are significantly elongated in one of the distinguished crystallographic in-plane directions (preferentially along [1$10] .{ }^{49,25}$

For single dash spectroscopy we used a microphotoluminescence setup equipped with a one-meter focal length monochromator, an InGaAs detector array, and a long working distance microscope objective with a numerical aperture of 0.4 to focus the excitation beam and collect the emission. The spectral resolution is estimated to 
about $20 \mu \mathrm{eV}$ for these measurements. Polarization-resolved analysis is performed using half-wave plate in front of horizontally aligned linear polarizer and the optical spectra for orthogonally polarized spectral lines can be resolved down to $\sim 5 \mu \mathrm{eV}$. The sample is excited nonresonantly under normal incidence by a continuous-wave laser diode emitting at $660 \mathrm{~nm}$, which is focused on the sample surface to approximately 2 - $\mu \mathrm{m}$-sized spot. The size of etched mesas is even smaller in order to further limit the number of probed nanostructures. The photoluminescence signal is collected backward and transmitted through the half-wave plate, a dichroic mirror and linear polarizer aligned for maximum efficiency of the diffraction grating. Such order provides the independence of the measured intensities of the setup polarization characteristics.

\section{RESULTS AND DISCUSSION}

\section{A. Luminescence}

Photoluminescence taken at $10 \mathrm{~K}$ from large ensemble of InAs/InGaAlAs/InP quantum dashes of three samples differing by the nominal amount of deposited InAs is presented in Fig. 2 showing the available spectral range for detection and analysis of single excitonic transitions. The tuning is in fact realized by mainly affecting the crosssectional size, ${ }^{12,50}$ and the resulting distribution of the emission shows the overlap with 1.3 and $1.55 \mu \mathrm{m}$, which is relevant from the application point of view in secure communication technologies.

An extracted microphotolumienscence spectrum of a single QDash is shown in Figure 3(a). It is taken on a structured mesa of $400 \times 400 \mathrm{~nm}^{2}$ which is sufficient for the observation of well separated optical transitions. ${ }^{4,14,51}$ In Figure 3a) we can see three spectral features around $990 \mathrm{meV}(\sim 1260 \mathrm{~nm})$ detected for three different excitation power conditions. The intensity dependence versus the excitation power is plotted in the inset of Fig. 3(a) in order to verify the slope of this dependence in the range of low excitation, which is close to linear for the lines at $994.6 \mathrm{meV}$ and $989.1 \mathrm{meV}$. The middle transition is not visible at low excitation and after increasing the excitation a superlinear increase is observed. The same spectra are shown in Fig. 3b) for two perpendicular linear polarizations aligned with respect to [110] and [1-10] directions taken from full-rotation polarization series. A splitting on the order of $50 \mu \mathrm{eV}$ for higher energy transitions and a lack of a splitting for the lowest energy line is observed. A vanishing splitting is expected for a charged exciton emission due to the canceled exchange interactions in case of a single particle residual spin of electron or hole, whereas the transition in the middle is assigned to a biexciton (XX) due to superlinear increase, and the high-energy line to exciton (X) by its noticeable fine structure splitting. In this scheme we can determine the spectral energy difference between exciton and biexciton of $-3.1 \mathrm{meV}$ and between exciton and charged exciton of $-5.7 \mathrm{meV}$, indicating the binding states for both, while $\mathrm{X}$ emission line is on higher energy side. No signature of any unbound state on the higher energy side of the spectrum has been identified.

The next illustration presented in Fig. 4 shows three other examples of excitonic complexes at various wavelengths of emission taken on the structured mesas. The origin of the lines has been determined in the same way as above confirming the neutral exciton, the biexciton and one bound trion. The energetic difference changes smoothly for the cases emitting at around $1212 \mathrm{~nm}, 1424 \mathrm{~nm}$ and $1552 \mathrm{~nm}$. Such spectra reveal a 
specific spectroscopic pattern for optically active single InAs QDashes, as also confirmed by the measurements of the second-order cross-correlation function presented elsewhere. ${ }^{3,4}$ Although for the case emitting close to $1552 \mathrm{~nm}$ the spectral density is relatively high, the other optical transitions have been verified as not correlated with identified excitonic complexes from a single quantum dash by the same methodology. Furthermore, as we found no signatures of any correlation with other spectral features it suggests that oppositely charged trion might be unattainable experimentally in this system.

\section{B. Binding energies and fine structure splitting}

The spectroscopic data has been collected for more than 50 single InAs QDashes in a way described in the previous section. The spectral range is limited to about $0.8 \mathrm{eV}$ on the lower energy side by a detection efficiency decrease of the InGaAs detector used in our experiments, whereas the maximal energy is $1.05 \mathrm{eV}$ and corresponds to the smallest available QDashes. In Figure 5 we present the determined binding energies of biexciton and trion in function of emission energy of exciton from the same QDash. According to the definition of binding energy $\Delta E\left(X^{*}\right)=E_{X^{*}}-E_{X}$, which is related to photon energies emitted by an excitonic complex $X^{*}$ and exciton $X$ given by $E_{X^{*}}$ and $E_{X}$, respectively, the value of $\Delta E\left(X^{*}\right)$ implies a bound (unbound) state when it is negative (positive) and $E_{X^{*}}$ is lower (higher) than $E_{X}$. One needs to be careful with such a notation (which, however, reflects better the physical situation in our opinion) when comparing to results of other papers where oppositely defined binding energies can also be used. As seen from Fig. 5, all the binding energies determined here remain on the negative side of the energy scale, therefore, sometimes we will refer to the absolute values. The size dependence in Fig. 5 is very smooth for the biexciton and its magnitude decreases slightly with increasing the energy of emission (binding energy decreases). The distribution appears to be narrower on the lower energy side where the binding energy is equal to about $-3.5 \mathrm{meV}$. It gets more spread when going to higher exciton energies, and can reach values from -2.5 to $-4 \mathrm{meV}$. This might be directly related to the intermixing effect with the InGaAlAs barrier, where smaller nanostructures may be more sensitive to small fluctuations of shape and composition (see the theoretical analysis in the next section). In case of the charged exciton, the distribution broadening effect is not pronounced. However, the emission energy dependence for the charged exciton shows a more evident increase of the binding energy with the emission energy (binding energy varies from $-4 \mathrm{meV}$ to $-5.8 \mathrm{meV}$. The gap in the data of Fig. 5 in the energy range between 0.9 and $0.95 \mathrm{eV}$ is related mainly to the experimental difficulties in dealing with a strong water-vapor-related absorption overlapping with relatively weak optical transitions.

Figure 6 shows the fine structure splitting of the bright exciton determined by the average of values obtained from the respective biexciton and exciton emissions polarized along [1-10] and [110] directions. The largest splitting amounts to approximately $190 \mu \mathrm{eV}$, which is, however, not the largest ever reported in spite of extraordinary asymmetry of the investigated system. ${ }^{28,31}$ What is noticed, there is no clear dependence on energy of emission, and instead a vast distribution of the fine structure splitting from below $50 \mu \mathrm{eV}$ to above $150 \mu \mathrm{eV}$ is observed for 0.8-0.9 eV energy range. Based on this observation we can conclude that the confinement potential is very random from QDash to QDash, possibly partly due to previously predicted and verified localized centers that might exist in the optically active structures within such nanostructure exhibiting irregularities in 
morphology. ${ }^{9}$ A much more regular behavior is seen in the emission range around $1 \mathrm{eV}$ where the FSS is typically in-between of $40-60 \mu \mathrm{eV}$ with few exceptions above this range. The conclusion is that the potential anisotropy related to the typical elongation of QDashes must be relevant leading to a stronger influence of the exchange interaction, while smaller splittings are possible due to geometrical (or compositional) fluctuations in the plane of some dashes, which might confine excitons in a smaller and less anisotropic volume. ${ }^{9}$

\section{Theoretical considerations}

We model the InAs QDashes as highly elongated wedges with triangular shaped cross-sections according to available morphological data (schematics shown in the inset of Fig. 7). We study a family of systems with the heights varying from 1.8 to $4.2 \mathrm{~nm}$ (i.e. 6 to 14 monolayers). In order to simulate the realistic tendencies where the characteristic sizes scale linearly ${ }^{12}$ we kept both the height to base width and the length to base width ratios constant and equal to 4 . Hence, the base width varies from 7.2 to $17 \mathrm{~nm}$ and the length from 28.8 to $68 \mathrm{~nm}$. Once the geometry of the system is established we calculate equilibrium atomic positions using the valence force field (VFF) model of Keating. ${ }^{54-56}$

In these calculations we have modeled QDashes using two different compositions, the first being pure InAs. However, the realistic nanostructures are always a subject of alloying and lattice randomness. Therefore, in the second approach we have also studied a system with the barrier material mixed into the QDash. In particular, we considered an intermixed case where the pure InAs QDash composition was replaced with (uniformly distributed) $\mathrm{In}_{0.9} \mathrm{Ga}_{0.05} \mathrm{Al}_{0.05} \mathrm{As}$ alloy. ${ }^{57}$ Additionally, we model the QDash and the surrounding InGaAlAs barrier using two distinct methods as well. In the first approach, we utilize virtual crystal approximation (VCA) where the VFF parameters are taken as a weighted average of the barrier material (GaAs, AlAs, InAs) parameters. In the second, fully atomistic approach, we treat the surrounding matrix (and the dash in the alloyed case) as a set of individual atoms with random compositions. We consider uniform composition profile resulting in the desired composition $\left(\mathrm{In}_{0.528} \mathrm{Ga}_{0.234} \mathrm{Al}_{0.238} \mathrm{As}\right.$ ). We also note that in several cases we performed (for comparison purpose) calculations for InAs QDashes embedded in pure InP barrier.

Once the atomic positions are found we calculate single particle states using an atomistic $\mathrm{sp}^{3} \mathrm{~d}^{5} \mathrm{~s}^{* 44,56,58,59}$ tightbinding model that accounts for d-orbitals and the spin-orbit interaction, and is capable to treat quaternary alloys such as InGaAlAs. Similarly, in order to calculate strain, we performed the electronic structure calculation using either the VCA or fully atomistic approach. In the VCA method we treat alloys by defining tight-binding parameters as weighted averaged of materials constituting the system. In this case, the valence band offset ${ }^{48,59}$ between InAs QDash and the mixed $\operatorname{In}_{0.528} \mathrm{Ga}_{0.234} \mathrm{Al}_{0.238}$ As matrix was set to $0.226 \mathrm{eV}$ (based on the band offset values from Ref. ${ }^{48}$ ). In the fully atomistic treatment, arsenic atoms in the quaternary alloy (as well as arsenic atoms on the interfaces) have their on-site parameters calculated as an average of the nearest neighbors. Finally, the single particle calculation is followed by the many-body configuration interaction ${ }^{56}$ calculation that produces the energy and optical spectra of excitons and excitonic complexes. In the configuration interaction we include all the possible determinants constructed from the 12 lowest-energy electron and 12 lowest-energy hole states 
(including spin). Finally, the optical spectra are found by calculating the oscillator strength for optical transition due to the recombination of one electron-hole pair in a given exciton state using the Fermi's golden rule. ${ }^{56}$

Figure 7 shows single exciton ground state energy as a function of the QDash height calculated using different atomistic approaches: VCA, fully atomistic (ATM) model, and fully atomistic approach with the alloyed quantum dash composition (ATM+Intermixing). As discussed earlier, the change of quantum dash height corresponds to the change of quantum dash all dimensions, i.e. base and length as well. Therefore, exciton energy shows a pronounced decrease from over $1.0 \mathrm{eV}$ to below $0.8 \mathrm{eV}$ with the increasing dimensions due to decrease of a quantum confinement. Similar results have been observed in the experiment as shown earlier. In general, the difference between the VCA and ATM approaches is on the order of $25 \mathrm{meV}$, with ATM results systematically lower than the VCA ones, we speculate that this is probably due to more efficient strain relaxation in the ATM model. On the other hand, energies given by the ATM model with the intermixing are typically larger by $\sim 0.1 \mathrm{eV}$ from ATM (without intermixing). This effect is expected consequence of incorporation of the wide-band gap barrier material into the quantum dash structure.

In order to study the energy spectra of QDashes we use the definition of the excitonic complex binding energy, which for a given complex is calculated with respect to the energy of the single (neutral) exciton as follows: $46,60,61$

$$
\begin{gathered}
\Delta E_{C I}(X X) \equiv E_{X X}-E_{X}=J_{e e}+J_{h h}-2 J_{e h}-\Delta \mathrm{E}_{\text {corr }}(X X)=\Delta E_{H F}(X X)-\Delta E_{c o r r}(X X), \\
\Delta E_{C I}\left(X^{-}\right) \equiv E_{X^{-}}-E_{X}=J_{h h}-J_{e h}-\Delta \mathrm{E}_{\mathrm{corr}}\left(X^{-}\right)=\Delta E_{H F}\left(X^{-}\right)-\Delta E_{c o r r}\left(X^{-}\right) .
\end{gathered}
$$

Herein CI index denotes values calculated by the full configuration interaction approximation as used in this work, $E_{X X}, E_{X}-$ and $E_{X}$ are biexciton, charged exciton and exciton recombination energies. The binding energies were further divided into two contributions. The first (HF) is the estimation of the binding energy in the spirit of Hartree-Fock (single configuration) approximation. The second (corr) is the correction due to correlations that accounts for effects of configuration mixing. Whereas inaccurate, the HF approach allows for simplified analysis, and it utilizes several Coulomb matrix elements only. These are namely $\mathrm{J}_{\mathrm{ee}}, \mathrm{J}_{\mathrm{eh}}$, and $\mathrm{J}_{\mathrm{hh}}$ which are correspondingly electron-electron, electron-hole, and hole-hole Coulomb integrals calculated for the electron (e) and hole (h) in their ground states.

Figure 8 shows these integrals calculated using three different approaches for the atomistic calculations, and for several QDashes of different shapes. We observe that the magnitude of these integrals grows with increasing values of the exciton energy. This is a hallmark of quantum confinement which is stronger for smaller QDashes with higher excitonic energy. The stronger confinement results in stronger wave-function overlap leading to a more pronounced electron-electron, electron-hole, and hole-hole interactions. Moreover, when going beyond the VCA model one observes fluctuations on top of clear monotonic trends: these are related to the lattice randomness. In the ATM case there are composition fluctuations in the barrier region only, where only tails of QDash wave-functions are localized. On the contrary, in ATM+Intermixing case, both the barrier and the QDash 
are subject to lattice randomness and hence there are more pronounced fluctuations of the Coulomb integrals values.

Apart from fluctuations, there is a common characteristic for all the three models: the hole-hole repulsion grows faster with the increasing confinement than the two other integrals. The relatively strong repulsion between holes could be understood in terms of their higher effective mass leading to stronger confinement than that of electrons and thus larger susceptibility to change in nanostructure dimensions. Additionally, the hole states are formed predominantly from the p-type atomic orbitals of highly-directional character and as such are affected not only by the hydrostatic strain, but also by the (highly anisotropic) biaxial strain. On the other hand the electrons are constituted by s-type atomic orbitals - they reveal no angular dependence and thus are affected by hydrostatic component of strain only. Therefore, the hole states should in principle be more susceptible to quantum dash anisotropy resulting from highly-elongated geometry. This anisotropy is particularly strong for smaller QDashes, where hole states are affected by strong lateral confinement in the [110] direction and weak confinement in the [1-10] direction. The increased hole-hole repulsion has further a pronounced effect on the excitonic spectra.

Next, we move to the energy spectra of excitonic complexes for variety of modeled structure geometries. Our calculations indicate that the positively charge exciton $\left(\mathrm{X}^{+}\right)$is strongly unbound, i.e. its energy is several meV larger than the $\mathrm{X}$ energy. Therefore, our theoretical results suggest that the CX complex observed experimentally should be identified as $\mathrm{X}^{-}$. Hence, in the following we focus our attention on $\mathrm{X}, \mathrm{X}^{-}$, and $\mathrm{XX}$ complexes. Figure 9 shows the biexciton and the negatively charged exciton ground state emission energies calculated with respect to the ground state energy of the single exciton, and presented as a function of the exciton energy. Importantly, all three models show characteristic trends with a pronounced $\mathrm{X}^{-}$binding energy dependence on the exciton energy (and thus effectively on QDash size), whereas the biexciton binding energy dependence remains relatively flat. Our calculations underestimate the absolute magnitude of excitonic complexes binding energies by about $1 \mathrm{meV}$ when compared to the experimental data, most likely due to the difference between shape and composition assumed in theory and those of the actual QDashes, which can however be hardly determined with any better precision. Additionally, in our CI approach as calculated in a limited basis (due to computational limitation), a further increase of the basis would likely lower the excitonic complexes binding energies. Moreover, any other more subtle effects as e.g. coupling to phonons which can also affect the binding energies ${ }^{62}$ have not been included. Nevertheless, our theoretical results reproduce well the characteristics as seen in the experiment.

Figure 10 shows the biexciton and the negatively charged exciton binding energies in function of the ground state energy of the neutral exciton, calculated by using both the VCA at the level of Hartree-Fock (HF) approximation and the configuration interaction (CI) model. The HF model predicts in this case an unbound biexciton (i.e. having positive binding energy). As the hole-hole repulsion increases with the enhancement of the confinement, the (positive) binding energy increases as well. Thus at the level of HF approximation the biexciton remains unbound in all cases, with the large positive binding energy growing with $\mathrm{X}$ energy and reaching up to 2 $\mathrm{meV}$ for the smallest considered system. With correlations accounted for by the CI method, the XX binding energies are considerably shifted down in the energy in the direction to negative values (correction varying from -2 to $-4 \mathrm{meV}$ ) and reveal quite a flat trend in the $\mathrm{X}$ energy, opposed to that predicted by the HF method only. In 
other words, QDashes are highly correlated systems, and thus the magnitude of correction due to correlations exceeds the contribution obtained by the rather simplified HF approximation.

Correlations have also a significant effect on the negatively charged exciton spectra changing its binding energy by approximately $-2 \mathrm{meV}$. Importantly however, the overall trend of the $\mathrm{X}^{-}$binding energy is quantitatively well reproduced already at the level of $\mathrm{HF}$ approximation: the $\mathrm{X}^{-}$binding energy grows with the $\mathrm{X}$ ground state energy. At the level of HF approximation this binding energy is given simply as a difference of electron-electron repulsion and electron-hole attraction $\left(\mathrm{J}_{\mathrm{ee}}-\mathrm{J}_{\mathrm{eh}}\right)$. As shown earlier in Fig. 8 the electron-hole $\mathrm{J}_{\mathrm{eh}}$ attraction grows faster with the confinement than electron-electron repulsion and hence results in the growth of $\mathrm{X}^{-}$binding energy with increasing confinement.

Finally, we move to the bright exciton fine structure splitting ${ }^{63}$ as shown in Fig. 11. Our theoretical results demonstrate that the FSS decreases with increasing X emission energy. In other words, the FSS increases with the overall quantum dash size. Such dependence of the anisotropic exchange interaction ${ }^{63}$ is opposite to that of the direct Coulomb interactions discussed above. The Coulomb direct terms could have been understood in terms of increased wavefunction overlap in a smaller, more confined nanostructure, whereas this apparently does not apply to the FSS. Traditionally, the FSS is associated with the nanostructure shape elongation ${ }^{35,63}$. This is however not exactly a case here, since in our simulation we use fixed lateral aspect ratio (4:1). Recent theoretical progress ${ }^{42,64-66}$ in understanding of the excitonic fine structure points at the anisotropy of the overall (lattice+shape) confinement potential, rather than shape elongation only. Thus, apart from the base elongation, the QDash anisotropy comes from the presence of atomic interfaces at the top four facets of a quantum dash: the larger (longer and taller) the QDash the larger the area of these facets and hence the more pronounced role of atomic interfaces related anisotropy resulting in the increased FSS.

The calculated reduction of the fine structure splitting with the $\mathrm{X}$ emission energy is in relatively good agreement with the experiment (Fig. 6): the FSS is reduced approximately by a factor of 4 , when going from 0.8 $\mathrm{eV}$ to $1 \mathrm{eV}$ of the exciton emission energy. We note however that there is yet another important factor affecting the FSS, which is intermixing and the related lattice randomness. From one point of view, intermixing increases the $\mathrm{X}$ emission energy as discussed earlier, on the other hand it also smears out the potential anisotropy ${ }^{42,67}$. In effect, already at $\mathrm{x}=0.05$ of barrier material intermixed into the QDash region (Fig. $11 \mathrm{c}$; ATM+Intermixing) the FSS is reduced by about $20-30 \mu \mathrm{eV}$, as compared to the case without intermixing (ATM). We have additionally calculated high intermixing level $\mathrm{x} \geq 0.10$ corresponding to $\mathrm{X}$ emission energy $\sim 1.1-1.2 \mathrm{eV}$ unobserved in the experiment. At this level of the intermixing the FSS is pushed down well below $40 \mu \mathrm{eV}$ and strongly varies from QDash to QDash. Thus apart from the FSS reduction, the intermixing introduces significant fluctuations of the FSS value. ${ }^{42}$ The role of lattice randomness is particularly visible in Fig. 12. In this figure, the exciton fine structure splitting was obtained by running calculations for 6 random realizations (samples) for each quantum dash size considered in the paper. Since we consider 5 different QDash sizes this gives total of 30 systems. Each sample corresponds to different local atomic arrangements, whereas the average composition remains constant in all samples (please note in particular Fig. 1 of the paper from Ref. ${ }^{46}$ ). Calculations were performed using the ATM+Intermixing model, where the $\mathrm{In}_{0.9} \mathrm{Ga}_{0.05} \mathrm{Al}_{0.05} \mathrm{As}$ QDash was embedded in the 
$\mathrm{In}_{0.528} \mathrm{Ga}_{0.234} \mathrm{Al}_{0.238} \mathrm{As}$ barrier. As shown in Fig. 12, the intermixing leads to a broad distribution of fine structure splitting values on top of general size dependent trends. Despite relatively simple, uniform composition profile assumed in our calculations, the predicted fine structure splitting values are in qualitative agreement with the experimental results (Fig. 6). We conclude that the atomistic calculations suggest that lattice randomness, as well as QDash shape and size variations are responsible for the broad distribution of FSS values observed in the experiment.

\section{CONCLUSIONS}

In this work we examined, both experimentally and theoretically, single QDash nanostructures emitting in a broad spectral range from $0.8 \mathrm{eV}$ to above $1 \mathrm{eV}$ made of InAs/InGaAlAs on InP. We obtained a satisfactory agreement between microphotoluminescence spectra and calculated energies by atomistic tight-binding approach using configuration interaction method. The data concerns the binding energy dependence of both the biexciton and the negatively charged exciton as well as the exciton fine structure splitting. Our results show that the electronic structure of this system is strongly influenced by Coulomb correlations which are reflected in the negative biexciton binding energy of about $-3.5 \mathrm{meV}$ with rather smooth energy dependence and even more bound negative trion of binding energy in range of -4.5 to $-5.5 \mathrm{meV}$. There is no evidence of the positively charged trion in the experiment due to a possible high concentration of excess carriers. By calculated energies of excitonic complexes we observed atypical increase of correlations for smaller QDashes which is related to the strain field that affects the charge spacings due to size change. The exciton fine structure splitting is experimentally largely distributed suggesting strong impact of the structural details. However, by performing calculations for fixed lateral aspect ratio of 4 we identified that increasing proportionally the nanostructure dimensions causes higher energy splittings reaching about $150 \mu \mathrm{eV}$ which is comparable to the measured values in the upper limit. By introducing the intermixing to the system we obtained smaller FSS suggesting that atomistic rearrangement between the QDash and the barrier structure might be a reason of the broad data distribution at a given spectral range. 


\section{ACKNOWLEDGEMENTS}

We would like to thank Paweł Machnikowski from the Division of Theoretical Physics, Wroclaw University of Science and Technology, for critical reading of the manuscript and his valuable comments. In addition, P.M., J.M. and G. S. acknowledge support from the grant of National Science Centre of Poland No. 2011/02/A/ST3/00152 (Maestro), whereas M.Z. acknowledges support from the Polish National Science Centre under grant No. 2015/18/E/ST3/005 (Sonata Bis). The experiments have partially been performed within the Wroclaw University of Science and Technology laboratory infrastructure financed by the Polish Ministry of Science and Higher Education Grant No. 6167/IA/119/2012. 


\section{REFERENCES}

${ }^{1}$ J.P. Reithmaier, A. Somers, S. Deubert, R. Schwertberger, W. Kaiser, A. Forchel, M. Calligaro, P. Resneau, O. Parillaud, S. Bansropun, M. Krakowski, R. Alizon, D. Hadass, A. Bilenca, H. Dery, V. Mikhelashvili, G. Eisenstein, M. Gioannini, I. Montrosset, T.W. Berg, M. Van Der Poel, J. Mørk, and B. Tromborg, J. Phys. D. Appl. Phys. 38, 2088 (2005).

2 J.P. Reithmaier, G. Eisenstein, and A. Forchel, Proc. IEEE 95, 1779 (2007).

${ }^{3}$ Ł. Dusanowski, M. Syperek, P. Mrowiński, W. Rudno-Rudziński, J. Misiewicz, A. Somers, S. Höfling, M. Kamp, J.P. Reithmaier, and G. Sęk, Appl. Phys. Lett. 105, 021909 (2014).

${ }^{4}$ Ł. Dusanowski, M. Syperek, W. Rudno-Rudziński, P. Mrowiński, G. Sk, J. Misiewicz, A. Somers, J.P. Reithmaier, S. Höfling, and A. Forchel, Appl. Phys. Lett. 103, 253113 (2013).

${ }^{5}$ P. Mrowiński, A. Musiał, A. Maryński, M. Syperek, J. Misiewicz, A. Somers, J.P. Reithmaier, S. Höfling, and G. Sęk, Appl. Phys. Lett. 106, 053114 (2015).

${ }^{6}$ M.B. Ward, M.C. Dean, R.M. Stevenson, A.J. Bennett, D.J.P. Ellis, K. Cooper, I. Farrer, C. a Nicoll, D.A. Ritchie, and A.J. Shields, Nat. Commun. 5, 3316 (2014).

${ }^{7}$ R.M. Stevenson, R.J. Young, P. Atkinson, K. Cooper, D.A. Ritchie, and A.J. Shields, Nature 439, 179 (2006).

${ }^{8}$ P. Mrowiński, K. Tarnowski, J. Olszewski, A. Somers, M. Kamp, S. Höfling, J.P. Reithmaier, W. Urbańczyk, J. Misiewicz, P. Machnikowski, and G. Sęk, Acta Phys. Pol. A 129, 48 (2016).

${ }^{9}$ A. Musiał, P. Kaczmarkiewicz, G. Sęk, P. Podemski, P. Machnikowski, J. Misiewicz, S. Hein, S. Höfling, and A. Forchel, Phys. Rev. B - Condens. Matter Mater. Phys. 85, 035314 (2012).

${ }^{10}$ P. Mrowiński, J. Appl. Phys. (n.d.).

${ }^{11}$ A. Lundskog, C.-W. Hsu, K. Fredrik Karlsson, S. Amloy, D. Nilsson, U. Forsberg, P. Olof Holtz, and E. Janzén, Light Sci. Appl. 3, e139 (2014).

${ }^{12}$ A. Sauerwald, T. Kümmell, G. Bacher, A. Somers, R. Schwertberger, J.P. Reithmaier, and A. Forchel, Appl. Phys. Lett. 86, 253112 (2005).

${ }^{13}$ T. Mensing, L. Worschech, Y. Ling, S. Kaiser, R. Schwertberger, J.P. Reithmaier, and A. Forchel, Phys. Status Solidi C Conf. 82, 1161 (2003).

${ }^{14}$ G. Şk, P. Podemski, A. Musiał, J. Misiewicz, S. Hein, S. Höfling, and A. Forchel, J. Appl. Phys. 105, 086104 (2009).

${ }^{15}$ E. Waks, K. Inoue, C. Santori, D. Fattal, J. Vučković, G.S. Solomon, and Y. Yamamoto, Nature 420, 762 (2002).

${ }^{16}$ E. Knill, R. Laflamme, and G.J. Milburn, Nature 409, 46 (2001).

${ }^{17}$ P. Kok, W.J. Munro, K. Nemoto, T.C. Ralph, J.P. Dowling, and G.J. Milburn, Rev. Mod. Phys. 79, 135 (2007).

${ }^{18}$ C.L. Salter, R.M. Stevenson, I. Farrer, C. a Nicoll, D.A. Ritchie, and A.J. Shields, Nature 465, 594 (2010).

${ }^{19}$ C. Santori, D. Fattal, J. Vučković, G.S. Solomon, and Y. Yamamoto, Nature 419, 594 (2002).

${ }^{20}$ J.M. Gérard, B. Sermage, B. Gayral, B. Legrand, E. Costard, and V. Thierry-Mieg, Phys. Rev. Lett. 81, 1110 (1998).

${ }^{21}$ E. Peter, P. Senellart, D. Martrou, A. Lemaître, J. Hours, J.M. Gérard, and J. Bloch, Phys. Rev. Lett. 95, 1 (2005).

${ }^{22}$ M.H. Baier, A. Malko, E. Pelucchi, D.Y. Oberli, and E. Kapon, Phys. Rev. B - Condens. Matter Mater. Phys. 73, 205321 (2006).

${ }^{23}$ R. Trotta, A. Polimeni, F. Martelli, G. Pettinari, M. Capizzi, L. Felisari, S. Rubini, M. Francardi, A. Gerardino, P.C.M. Christianen, and J.C. Maan, Adv. Mater. 23, 2706 (2011).

${ }^{24}$ V. Baumann, F. Stumpf, C. Schneider, S. Kremling, L. Worschech, A. Forchel, S. Höfling, and M. Kamp, Appl. Phys. Lett. 100, 091109 (2012).

${ }^{25}$ L. Seravalli, C. Bocchi, G. Trevisi, and P. Frigeri, J. Appl. Phys. 108, (2010).

${ }^{26}$ J. Kettler, M. Paul, F. Olbrich, K. Zeuner, M. Jetter, P. Michler, M. Florian, C. Carmesin, and F. Jahnke, Phys. Rev. B 94, 045303 (2016).

${ }^{27}$ G. Saint-Girons, N. Chauvin, a Michon, G. Patriarche, G. Beaudoin, G. Bremond, C. Bru-Chevallier, and I. 
Sagnes, Appl. Phys. Lett. 88, 133101 (2006).

${ }^{28}$ N. Chauvin, B. Salem, G. Bremond, G. Guillot, C. Bru-Chevallier, and M. Gendry, J. Appl. Phys. 100, 073702 (2006).

${ }^{29}$ N. Chauvin, E. Tranvouez, G. Bremond, G. Guillot, C. Bru-Chevallier, E. Dupuy, P. Regreny, M. Gendry, and G. Patriarche, Nanotechnology 17, 1831 (2006).

${ }^{30}$ N.I. Cade, H. Gotoh, H. Kamada, H. Nakano, S. Anantathanasarn, and R. Nötzel, Appl. Phys. Lett. 89, 181113 (2006).

${ }^{31}$ N.I. Cade, H. Gotoh, H. Kamada, H. Nakano, and H. Okamoto, Phys. Rev. B - Condens. Matter Mater. Phys. 73, 1 (2006).

${ }^{32}$ V. V. Belykh, A. Greilich, D.R. Yakovlev, M. Yacob, J.P. Reithmaier, M. Benyoucef, and M. Bayer, Phys. Rev. B 92, 165307 (2015).

${ }^{33}$ V. Mlinar, M. Bozkurt, J. Ulloa, M. Ediger, G. Bester, A. Badolato, P. Koenraad, R.J. Warburton, and A. Zunger, Phys. Rev. B 80, 1 (2009).

${ }^{34}$ S. Rodt, A. Schliwa, K. Pötschke, F. Guffarth, and D. Bimberg, Phys. Rev. B 71, 155325 (2005).

${ }^{35}$ R. Seguin, A. Schliwa, S. Rodt, K. Potschke, U.W. Pohl, and D. Bimberg, Phys. Rev. Lett. 95, 257402 (2005).

${ }^{36}$ W. Langbein, P. Borri, U. Woggon, V. Stavarache, D. Reuter, and a. D. Wieck, Phys. Rev. B - Condens. Matter Mater. Phys. 70, 1 (2004).

${ }^{37}$ J.J. Finley, D. Mowbray, M. Skolnick, A. Ashmore, C. Baker, A. Monte, and M. Hopkinson, Phys. Rev. B 66, 153316 (2002).

${ }^{38}$ C. Jarlov, P. Gallo, M. Calic, B. Dwir, A. Rudra, and E. Kapon, Appl. Phys. Lett. 101, 191101 (2012).

${ }^{39}$ A. Schliwa, M. Winkelnkemper, and D. Bimberg, Phys. Rev. B 79, 075443 (2009).

${ }^{40}$ L. He, M. Gong, C.-F. Li, G.-C. Guo, and A. Zunger, Phys. Rev. Lett. 101, 157405 (2008).

${ }^{41}$ M. Abbarchi, C. Mastrandrea, T. Kuroda, T. Mano, K. Sakoda, N. Koguchi, S. Sanguinetti, a. Vinattieri, and M. Gurioli, Phys. Rev. B 78, 125321 (2008).

${ }^{42}$ M. Zieliński, Phys. Rev. B - Condens. Matter Mater. Phys. 88, 155319 (2013).

${ }^{43}$ R. Singh and G. Bester, Phys. Rev. B 84, 241402(R) (2011).

${ }^{44}$ M. Zieliński, Phys. Rev. B 86, 115424 (2012).

${ }^{45}$ J.W. Luo and A. Zunger, Phys. Rev. B - Condens. Matter Mater. Phys. 84, 17 (2011).

${ }^{46}$ M. Zieliński, K. Gołasa, M.R. Molas, M. Goryca, T. Kazimierczuk, T. Smoleński, A. Golnik, P. Kossacki, a. a. L. Nicolet, M. Potemski, Z.R. Wasilewski, and A. Babiński, Phys. Rev. B 91, 085303 (2015).

${ }^{47}$ H. Dery, E. Benisty, A. Epstein, R. Alizon, V. Mikhelashvili, G. Eisenstein, R. Schwertberger, D. Gold, J.P. Reithmaier, and A. Forchel, J. Appl. Phys. 95, 6103 (2004).

${ }^{48}$ I. Vurgaftman, J.R. Meyer, and L.R. Ram-Mohan, J. Appl. Phys. 89, 5815 (2001).

${ }^{49}$ K. Nishi, H. Saito, S. Sugou, and J.S. Lee, Appl. Phys. Lett. 74, 1111 (1999).

50 A. Somers, W. Kaiser, J. P. Reithmaier, A. Forchel, M. Gioaninni and I. Montrosset, Appl. Phys. Lett. 89, 061107 (2006)

${ }^{51}$ P. Mrowiński, A. Musiał, G. Sęk, J. Misiewicz, S. Höfling, A. Somers, S. Hein, and A. Forchel, Acta Phys. Pol. A 124, 801 (2013).

52 Y. Masumoto, S. Yoshida, M. Ikezawa, S. Tomimoto, and Y. Sakuma, Appl. Phys. Lett. 98, 061905 (2011).

${ }^{53}$ F. Ding, R. Singh, J.D. Plumhof, T. Zander, V. Křápek, Y.H. Chen, M. Benyoucef, V. Zwiller, K. Dörr, G. Bester, A. Rastelli, and O.G. Schmidt, Phys. Rev. Lett. 104, 067405 (2010).

${ }^{54}$ P.N. Keating, Phys. Rev. 145, 637 (1966).

${ }^{55}$ R.M. Martin, Phys. Rev. B 1, 4005 (1970).

${ }^{56}$ M. Zieliński, M. Korkusiński, and P. Hawrylak, Phys. Rev. B - Condens. Matter Mater. Phys. 81, 1 (2010).

${ }^{57}$ G. Sęk, K. Ryczko, M. Motyka, J. Andrzejewski, K. Wysocka, J. Misiewicz, L.H. Li, A. Fiore, and G. Patriarche, J. Appl. Phys. 101, (2007).

${ }^{58}$ J.-M. Jancu, R. Scholz, F. Beltram, and F. Bassani, Phys. Rev. B 57, 6493 (1998). 
${ }^{59}$ M. Zieliński, J. Phys. Condens. Matter 25, 465301 (2013).

${ }^{60}$ M. Zieliński, Nanoscale Res. Lett. 7, 265 (2012).

${ }^{61}$ G. a. Narvaez, G. Bester, and A. Zunger, Phys. Rev. B - Condens. Matter Mater. Phys. 72, 245318 (2005).

${ }^{62}$ P. Machnikowski, Phys. Rev. B - Condens. Matter Mater. Phys. 83, 1 (2011).

${ }^{63}$ M. Bayer, G. Ortner, O. Stern, A. Kuther, A. Gorbunov, A. Forchel, P. Hawrylak, S. Fafard, K. Hinzer, T. Reinecke, S.N. Walck, J.P. Reithmaier, F. Klopf, and F. Schäfer, Phys. Rev. B 65, 195315 (2002).

${ }^{64}$ R. Singh and G. Bester, Phys. Rev. B 88, 075430 (2013).

${ }^{65}$ M.A. Dupertuis, K.F. Karlsson, D.Y. Oberli, E. Pelucchi, A. Rudra, P.O. Holtz, and E. Kapon, Phys. Rev. Lett. 107, 1 (2011).

${ }^{66}$ K.F. Karlsson, M. a. Dupertuis, D.Y. Oberli, E. Pelucchi, a. Rudra, P.O. Holtz, and E. Kapon, Phys. Rev. B 81, 161307 (2010).

${ }^{67}$ A.I. Tartakovskii, M.N. Makhonin, I.R. Sellers, J. Cahill, A.D. Andreev, D.M. Whittaker, A.M. Fox, D.J. Mowbray, M.S. Skolnick, K.M. Groom, M.J. Steer, H.Y. Liu, and M. Hopkinson, Phys. Rev. B 193303, 1 (2004).

\section{Figures:}

a)

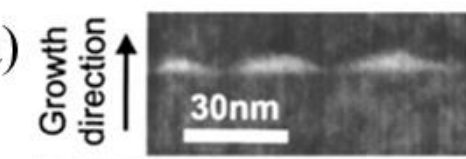

b)

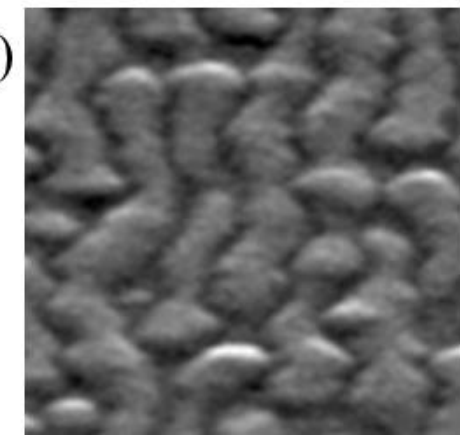

Figure 1. a) STEM micrograph of cross-sectional sizes of single QDashes (after ref. ${ }^{12}$ ) showing a triangular geometry, and b) SEM image of the uncapped QDash layer.

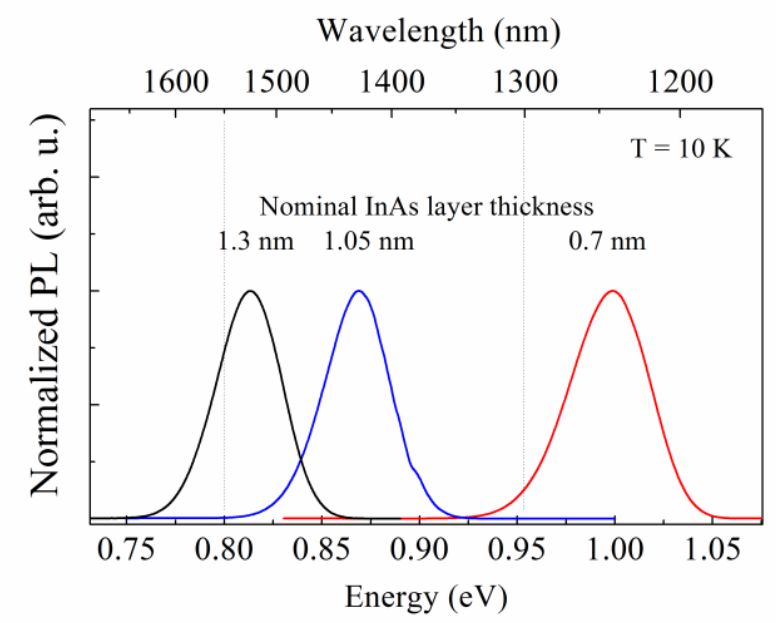

Figure 2. Photoluminescence emission from quantum dash ensemble for different nominal InAs layer thicknesses overlapping with the $2^{\text {nd }}(1300 \mathrm{~nm})$ and $3^{\text {rd }}(1550 \mathrm{~nm})$ telecommunication low loss windows. 

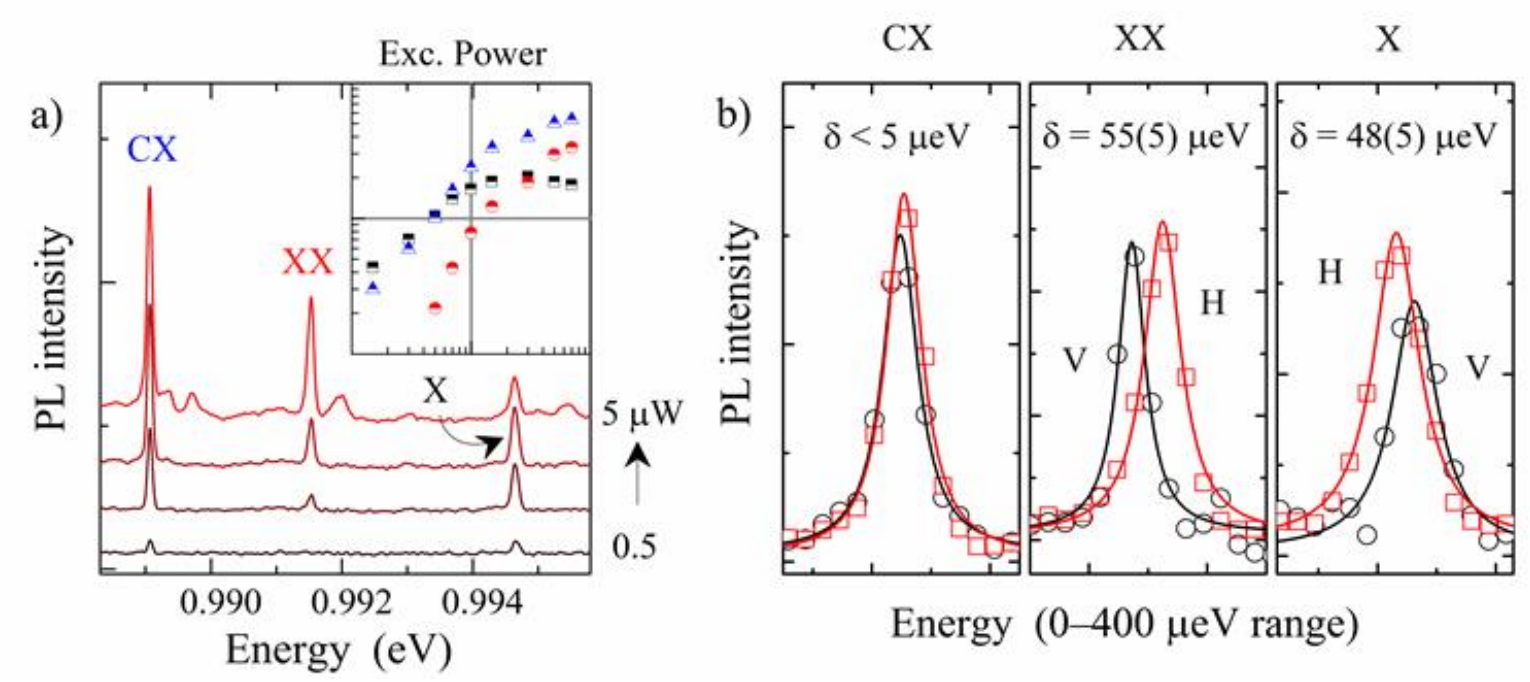

Figure 3. a) Microphotoluminescence spectra for various excitation powers from 0.5 to $5 \mu \mathrm{W}$. The inset shows the intensity dependence with a slope variation depending on the spectral feature. b) Polarization resolved spectra showing fine structure splitting indicating exciton and biexciton emission and no splitting for charged exciton.

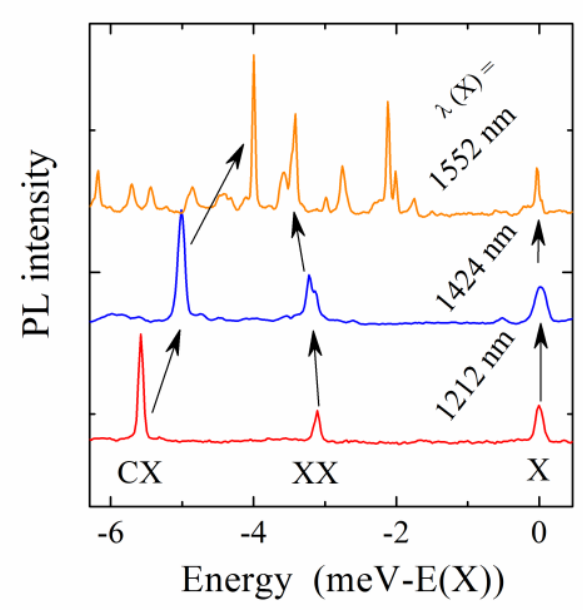

Figure 4. Excitonic complexes for different emission wavelengths showing alike spectroscopic pattern for biexciton and trion transitions. 


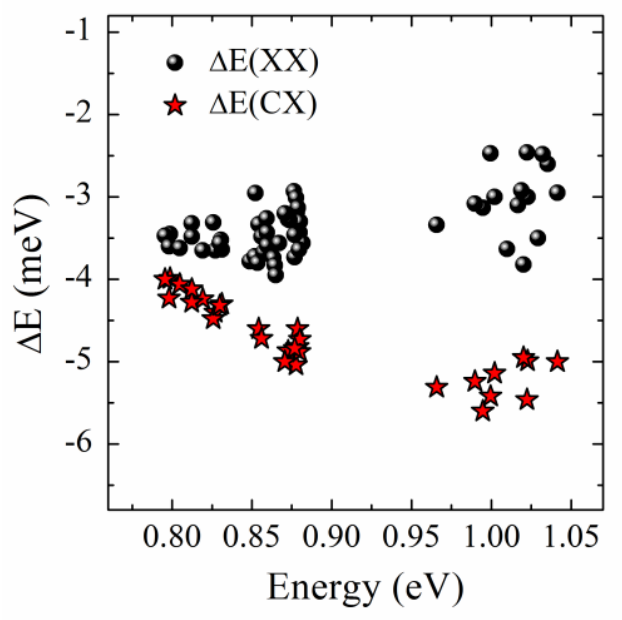

Figure 5. Binding energy of biexciton (circle) and charged exciton (star) state determined for ca 50 single QDashes in dependence of exciton emission energy.

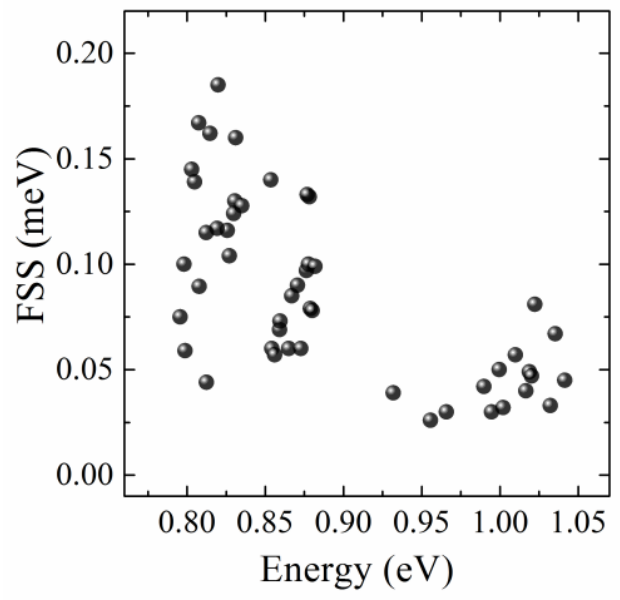

Figure 6. Exciton fine structure splitting averaged from biexciton and exciton transition in emission energy dependence. 


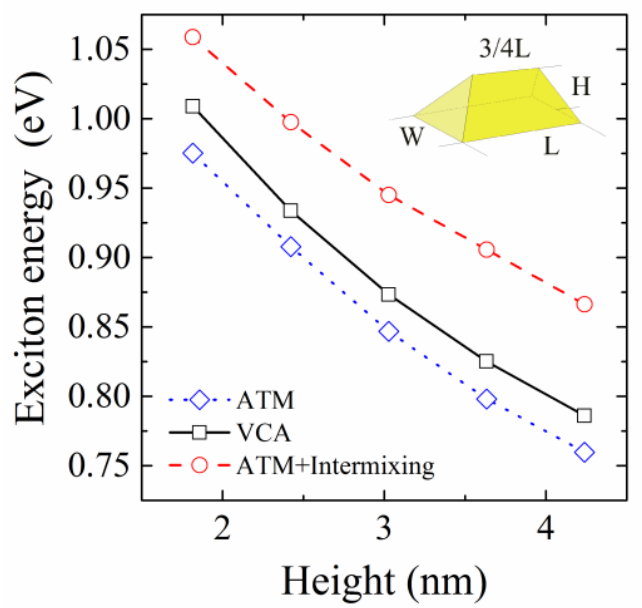

Figure 7. Exciton ground state energy calculated using different atomistic approaches as a function of quantum dash size $(\mathrm{H}-$ Height, $\mathrm{W}=4 \times \mathrm{H}, \mathrm{L}=4 \times \mathrm{W})$.
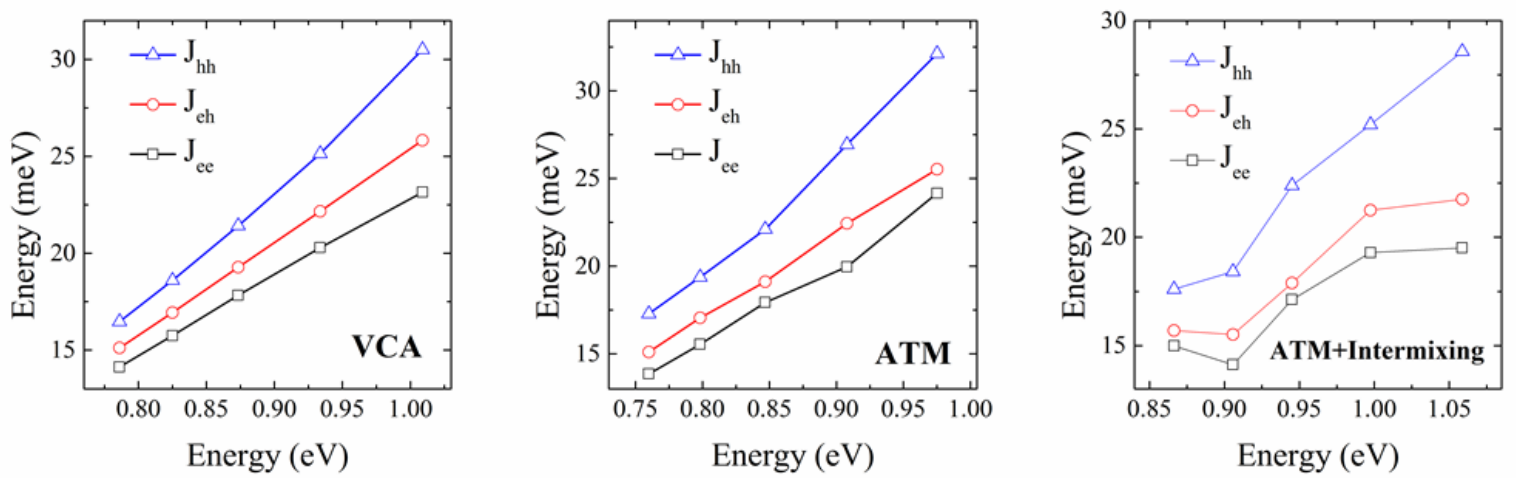

Figure 8. Electron-electron $\left(\mathrm{J}_{\mathrm{ee}}\right)$, electron-hole $\left(\mathrm{J}_{\mathrm{eh}}\right)$, and hole-hole $\left(\mathrm{J}_{\mathrm{hh}}\right)$ Coulomb integrals for electron and hole occupying their ground states calculated for several QDashes of different energy, and using different atomistic approaches.
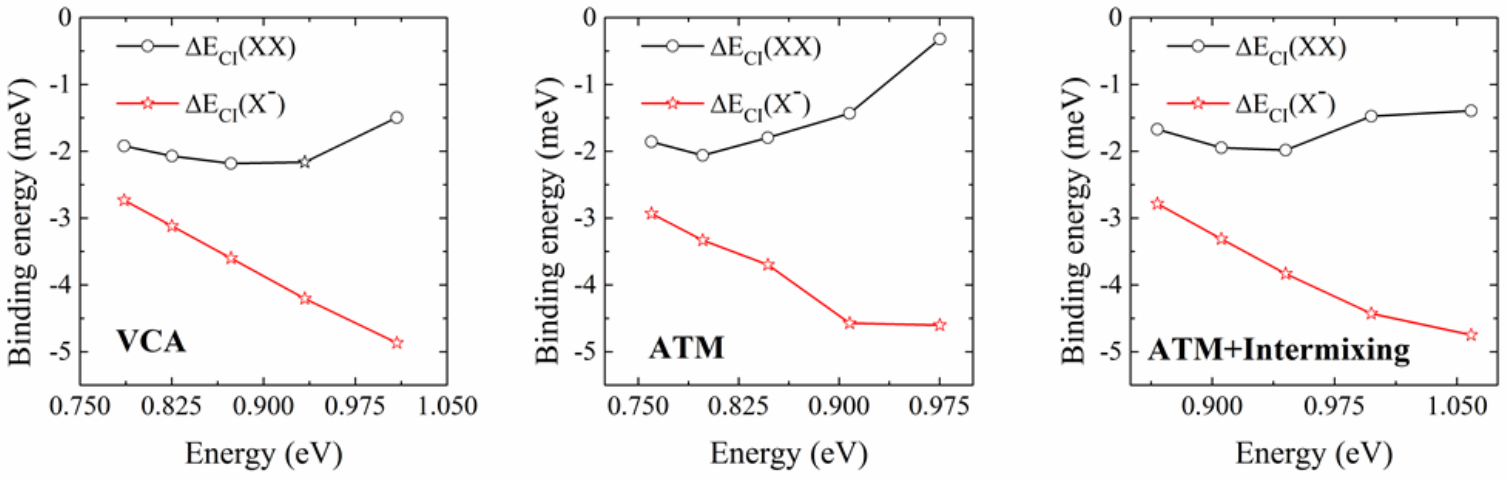

Figure 9. Binding energies of biexciton (circles) and charged exciton (stars) calculated using configuration interaction model and different atomistic approaches (see the text) as a function of exciton emission energy. 


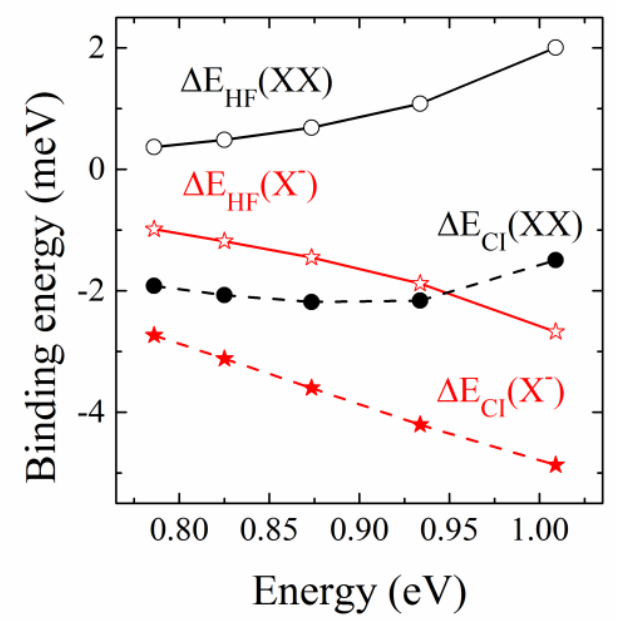

Figure 10. Negatively charged exciton $\Delta \mathrm{E}\left(\mathrm{X}^{-}\right)$and the biexciton $\triangle \mathrm{E}(\mathrm{XX})$ binding energies in Hartree-Fock $(\mathrm{HF})$ and configuration interaction (CI) pictures; calculated using VCA (see the text).
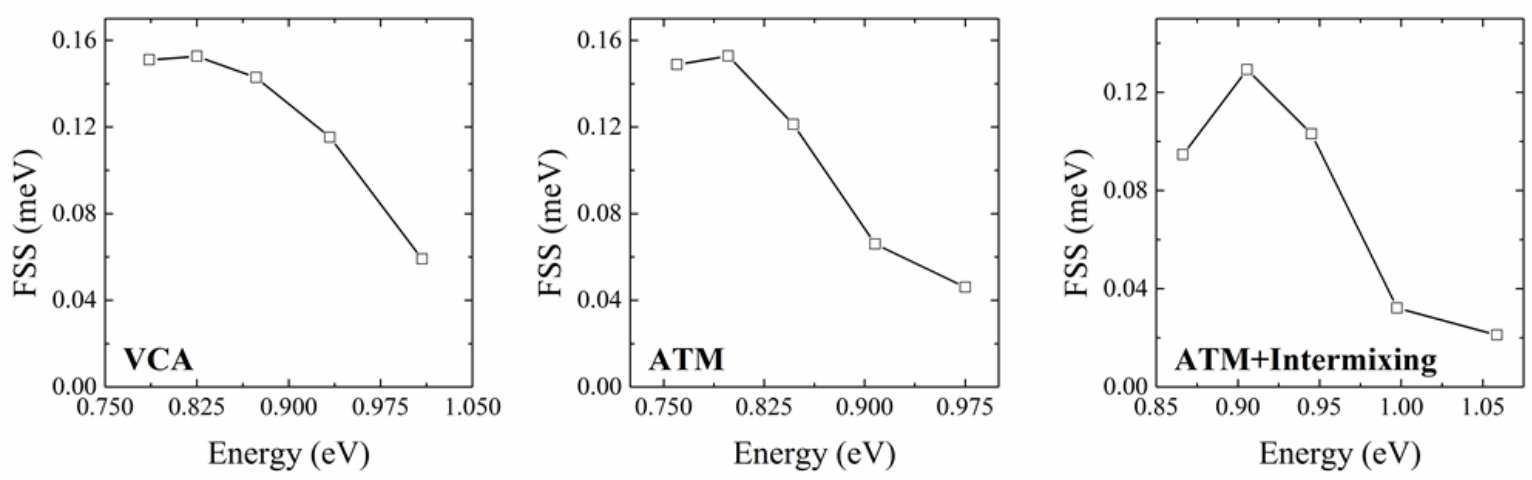

Figure 11. Exciton fine structure splitting calculated using different atomistic approaches (see the text) as a function of exciton emission energy.

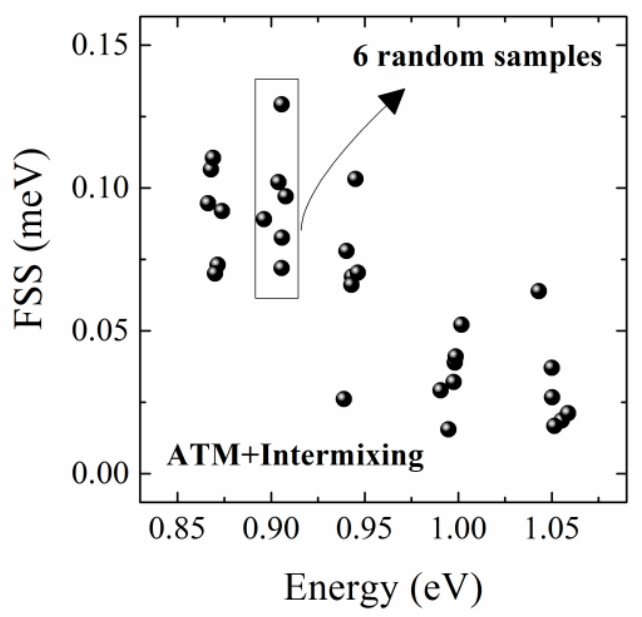

Figure 12. Exciton fine structure splitting calculated to illustrate the role of lattice randomness. These results were obtained by putting together 6 random realizations (samples) for each quantum dash size ( 5 different sizes) considered in the paper, giving total of 30 systems. Each sample corresponds to different local atomic 
arrangements, whereas the average composition remains constant in all samples. ${ }^{46}$ Calculations were performed using ATM+Intermixing model, where $\operatorname{In}_{0.9} \mathrm{Ga}_{0.05} \mathrm{Al}_{0.05} \mathrm{As}$ QDash was embedded in $\operatorname{In}_{0.528} \mathrm{Ga}_{0.234} \mathrm{Al}_{0.238} \mathrm{As}$ barrier. 